\title{
Spectrum of the open QCD flux tube in $d=2+1$ and its effective string description
}

\author{
Bastian B. Brandt* \\ Institute for Theoretical Physics, University of Regensburg, \\ 93040 Regensburg, Germany \\ E-mail: bastian.brandtephysik. uni-regensburg.de
}

\begin{abstract}
Simulations in lattice gauge theory suggest that the formation of a flux tube between quark and antiquark leads to quark confinement. It is conjectured that the infrared behaviour of the flux tube is governed by an effective string theory and simulations show good agreement between lattice data and its predictions. To next-to leading order $\left(R^{-3}\right)$ in the inverse $q \bar{q}$ separation $R$ the effective string theory is equivalent to Nambu-Goto string theory. For the open flux tube in three dimensions corrections appear at order $R^{-4}$. We compare these predictions to high-accuracy measurements of the groundstate energy of the flux tube in $3 \mathrm{~d} S U(2)$ and $S U(3)$ gauge theory and extract the coefficient of the leading order boundary term in the effective action.
\end{abstract}

The European Physical Society Conference on High Energy Physics

18-24 July, 2013

Stockholm, Sweden

\footnotetext{
*Speaker.
} 


\section{Introduction}

Lattice simulations provide strong evidence that the formation of a flux tube between a static quark and antiquark is a valid mechanism to describe the confinement of quarks. In the ultraviolet it is conjectured that the dynamics of the flux tube is well described by an effective string theory [1, $2,3,4]$. A simple ansatz for the woldsheet field theory is the action of a free bosonic string, the Nambu-Goto (NG) action with the spectrum [5]

$$
E_{n}^{\mathrm{NG}}(R)=\sigma R \sqrt{1+\frac{2 \pi}{\sigma R^{2}}\left(n-\frac{1}{24}(d-2)\right)}
$$

Here $\sigma$ is the string tension and $R$ the $q \bar{q}$ separation. In $d \neq 26$ the quantisation of the NG action suffers from the well known Weyl anomaly [1], rendering the interpretation of the NG action as the low energy effective action for the flux tube in the ultraviolet region inconsistent.

This consistency problem has triggered the development of alternative frameworks to write down the effective string action. Historically there are two main methods, the ansatz by Polchinski and Strominger [6] and the one by Lüscher and Weisz [7, 8] and for a long time it was unknown how to connect these two. Recently Aharony and collaborators performed extensive calculations in both theories, showing that the spectrum is equivalent in the two theories up to $\mathscr{O}\left(R^{-5}\right)$ and that it includes corrections to the NG spectrum [9, 10, 11, 12]. Furthermore, they provided a relation between the two methods based on a generalisation of the AdS/CFT correspondence for pure gauge theory where the confining string is a weakly coupled fundamental string moving in a weakly curved background [13]. Within this framework the two approaches correspond to the 'conformal' or 'static/unitary' gauge, respectively. Alternative methods to calculate the spectrum of the confining string have also been proposed recently [14, 15], leading to compatible predictions.

Simulations in pure gauge theories ${ }^{1}$ show striking agreement with the NG predictions for the energy levels of the open and the closed flux tube, as well as for the width of the flux tube at its midpoint. The accordance persists down to surprisingly small values of the $q \bar{q}$ separation where the flux tube cannot be expected to be shaped like a string. Deviations from NG became visible only recently with increasing precision in the measurements of the energy levels. For the open string spectrum these deviations are well described by the predictions from the effective string theory [22, 23]. Similar agreement has also been found concerning the predictions at finite temperature [24, 25] and for other objects in gauge theories with an effective string description (e.g. [26] and references therein).

In this proceedings article we present new results concerning the coefficient of the leading order correction to the NG spectrum of the open string. We begin by summarising the predictions from the effective string theory before we discuss briefly the details of the simulations and the extraction of the flux tube spectrum. We close with the discussion of the results for the boundary coefficient.

\footnotetext{
${ }^{1}$ For results see $[16,17]$ (open spectrum), $[18,19,20]$ (closed spectrum), [21] (width) and the included lists of references.
} 


\begin{tabular}{cc|cccc|ccccc}
$N$ & Lat & $\beta$ & $r_{0} / a$ & $\sqrt{\sigma} a$ & $\sqrt{\sigma} r_{0}$ & size & $R / a$ & $t_{s}$ & $n_{t}$ & \#meas \\
\hline \hline 2 & A & 5.0 & $3.9472(4)$ & $0.31212(7)$ & $1.2320(3)$ & $48^{3}$ & $2-12$ & 2 & 30000 & 1600 \\
& B & 7.5 & $6.2863(7)$ & $0.19630(4)$ & $1.2340(3)$ & $64^{3}$ & $2-26$ & 4 & 30000 & 1400 \\
& $\mathrm{C}$ & 10.0 & $8.5992(6)$ & $0.14354(2)$ & $1.2343(2)$ & $96^{3}$ & $2-30$ & 6 & 30000 & 2200 \\
\hline 3 & $\mathrm{~F}$ & 14.0 & $4.4433(3)$ & $0.27682(4)$ & $1.2300(2)$ & $48^{3}$ & $2-13$ & 2 & 20000 & 1900 \\
& $\mathrm{G}$ & 20.0 & $6.7073(4)$ & $0.18367(2)$ & $1.2319(2)$ & $60^{3}$ & $3-27$ & 4 & 20000 & 2300 \\
\hline \hline
\end{tabular}

Table 1: List of simulation parameters. $R$ denotes the $q \bar{q}$ separation, $t_{s}$ is the temporal extent of the LüscherWeisz sublattices and $n_{t}$ denotes the number of sublattice updates.

\section{Spectrum of the open confining string}

In the fundamental effective string theory, discussed in detail in [13], the full NG action is the only 'weight zero' 2 term entering the action, so that all additional terms in the spectrum will appear with respect to eq. (1.1). For the open string the first correction that appears is a four-derivative boundary term [9]. The associated energy levels are given by [10]

$$
E_{n}^{\mathrm{BC}}(R)=E_{n}^{\mathrm{NG}}(R)-\bar{b}_{2} \frac{\pi^{3}}{\sqrt{\sigma^{3}} R^{4}}\left(4 \bar{N}_{n}+\frac{d-2}{60}\right),
$$

where $\bar{N}_{n}$ is a factor that depends on the eigenstate at energy level $n$ (for a classification of the states via charge conjugation $\mathrm{C}$ and parity $\mathrm{P}, \bar{N}_{n}=\bar{N}_{n}^{\mathrm{CP}}$ and $\bar{N}_{0}=0, \bar{N}_{1}=1, \bar{N}_{2}^{++}=2$ and $\bar{N}_{2}^{--}=8$; see [22]) and $\bar{b}_{2} \equiv \sqrt{\sigma^{3}} b_{2}$ is a dimensionless non-universal parameter. The same result has also been found using an alternative ansatz [23]. The next correction is a regular (non-boundary) term appearing at $\mathscr{O}\left(R^{-5}\right)$ and can be expected to be non-universal as well. However, in $d=2+1$, the case considered in this study, it vanishes identically.

\section{Simulation details}

We perform simulations of pure $S U(2)$ and $S U(3)$ gauge theory in $2+1$ dimensions using the standard combination of heatbath and overrelaxation updates. The simulation parameters and lattice sizes are listed in table 1 .

The focus is on the groundstate potential that can be extracted with high precision using Polyakov loop correlation functions. For those correlators excited states are suppressed exponentially with $T$ so that their contribution to the groundstate signal can usually be neglected. In the following the energies are extracted via

$$
V(R) \equiv E_{0}(R)=-\frac{1}{T} \ln \left[\left\langle P^{*}(R) P(0)\right\rangle\right],
$$

where $T$ is the temporal extent of the lattice. The scale is set via the string tension as defined by the potential from eq. (2.1). For completeness we also list the result for the Sommer scale and $\sqrt{\sigma} r_{0}$ in table 2. The Polyakov loop correlation functions have been measured using the Lüscher-Weisz multilevel algorithm [27] with the parameters given in table 1. We have checked that finite size effects as discussed in [22] are small for all spatial extents $R$. The error analysis is done using the jackknife method with 100 bins for all lattices. We have also checked that varying the number of bins did not change the error estimates.

\footnotetext{
${ }^{2}$ In this framework the 'weight' counts the number of derivatives in the action. The induced metric, with two derivatives, has zero weight per definition.
} 


\begin{tabular}{c|cccc} 
Lat & $R_{\min } / a$ & $\bar{b}_{2}$ & $a V_{0}$ & $\chi^{2} /$ dof \\
\hline \hline A & 4 & $-0.0197(11)(52)$ & $0.2148(2)(3)$ & 0.1 \\
B & 5 & $-0.0210(4)(16)$ & $0.1740(1)(2)$ & 0.1 \\
C & 8 & $-0.0231(1)(22)$ & $0.14500(4)(11)$ & 0.2 \\
\hline F & 4 & $-0.0137(4)(10)$ & $0.2239(1)(2)$ & 0.02 \\
G & 6 & $-0.0170(3)(1)$ & $0.18166(5)(1)$ & 0.02 \\
\hline \hline
\end{tabular}

Table 2: Results for the fitparameters as explained in the text.
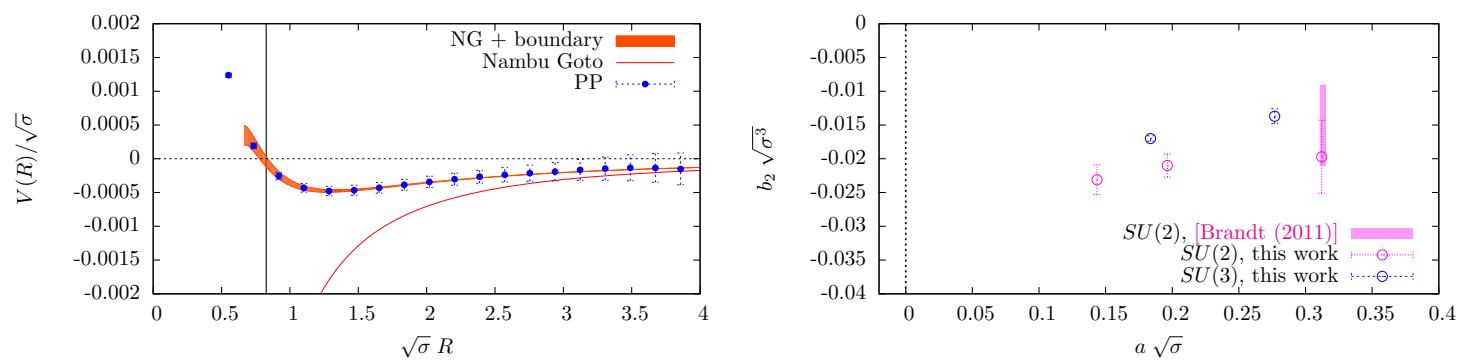

Figure 1: Left: Results for the groundstate $q \bar{q}$ potential on lattice G, normalised as in [22]. The orange band is the fit result and the vertical line indicates the fitrange. Right: Results for $\bar{b}_{2}$ versus the lattice spacing in units of the string tension in comparison to the result from [22].

\section{Extraction of the boundary coefficient}

The boundary coefficient has been extracted from the groundstate energies by a fit to the form given in eq. (2.1) with $\sigma, \bar{b}_{2}$ and an additive normalisation constant $V_{0}$ as free parameters. The lower cut $R_{\min }$ has been chosen to be the second smallest $q \bar{q}$ separation for which the fit still provided $\chi^{2} /$ dof values smaller than one and is listed in table 2 together with the results for $\bar{b}_{2}$ and $V_{0} . \sigma$ is given in table 1 . The systematic error associated with this particular choice (the second error for the quantities in table 2) is estimated from the spread of the results obtained by fits with a minimal $q \bar{q}$ separation $R_{\min } \pm 1$. Figure 1 (left) shows the result for the groundstate potential of lattice $\mathrm{G}$ in comparison to the fit and the NG prediction. The plot displays the excellent agreement between the predictions and the data.

In the case of $S U(2)$ the error of $\bar{b}_{2}$ is dominated by the systematic uncertainty. This is different for $S U$ (3), where in general much less dependence on $R_{\min }$ is seen. Furthermore, $\chi^{2} /$ dof differs by an order of magnitude between the two cases. A possible explanation is provided by the fact that the picture of a single non-interacting flux tube is consistent only in the limit $N \rightarrow \infty$. This suggests that we observe a remnant of the interaction of the flux tube with the lightest glueballs which perturbs the agreement with the effective string predictions (see also the discussions in [19, 28, 29]).

In figure 1 (right) the results for $\bar{b}_{2}$ are plotted versus the lattice spacing in units of the string tension. The dependence on the lattice spacing is relatively mild in both cases and the results are close together, indicating only a small $N$-dependence of $\bar{b}_{2}$. Whether this is also true in the continuum remains to be seen. A detailed continuum extrapolation together with comparisons to data for the excited states will be presented in a future publication.

It would be interesting to compare to the results from $Z(2)$ gauge theory [23]. This is difficult, since in [23] the coefficient $\bar{b}_{2}$ has been extracted using the expansion of the NG energies to $\mathscr{O}\left(R^{-3}\right)$ instead of the full resumed energies. Note, that extracting $\bar{b}_{2}$ from an expansion of eq. (1.1) to 
different orders in $1 / R$ even leads to different signs for $\bar{b}_{2}$, since the signs of the expansion terms alternate. Following the discussion in section 2 and in [10, 19], starting from eq. (2.1) thus appears to be a proper and unambiguous choice for the extraction of $\bar{b}_{2}$.

Acknowledgments: I would like to thank M. Panero for the invitation to give this talk and M. Caselle for discussions. The simulations were done in parts on the LC2 cluster at the university of Mainz and on the computing resources of the university of Regensburg.

\section{References}

[1] P. Goddard et al, Nucl. Phys. B 56 (1973) 109.

[2] Y. Nambu, Phys. Lett. B 80 (1979) 372.

[3] M. Lüscher, K. Symanzik and P. Weisz, Nucl. Phys. B 173 (1980) 365.

[4] A. M. Polyakov, Nucl. Phys. B 164 (1980) 171.

[5] J. F. Arvis, Phys. Lett. B 127 (1983) 106.

[6] J. Polchinski and A. Strominger, Phys. Rev. Lett. 67 (1991) 1681.

[7] M. Lüscher and P. Weisz, JHEP 0207 (2002) 049 [hep-lat/0207003].

[8] M. Lüscher and P. Weisz, JHEP 0407 (2004) 014 [hep-th/0406205].

[9] O. Aharony and E. Karzbrun, JHEP 0906 (2009) 012 [arXiv:0903.1927 [hep-th]].

[10] O. Aharony and N. Klinghoffer, JHEP 1012 (2010) 058 [arXiv:1008.2648 [hep-th]].

[11] O. Aharony and M. Field, JHEP 1101 (2011) 065 [arXiv:1008.2636 [hep-th]].

[12] O. Aharony, M. Field and N. Klinghoffer, JHEP 1204 (2012) 048 [arXiv:1111.5757 [hep-th]].

[13] O. Aharony and Z. Komargodski, JHEP 1305 (2013) 118 [arXiv:1302.6257 [hep-th]].

[14] S. Dubovsky, R. Flauger and V. Gorbenko, JHEP 1209 (2012) 044 [arXiv:1203.1054 [hep-th]].

[15] M. Caselle et al, JHEP 1307 (2013) 071 [arXiv:1305.1278 [hep-th]].

[16] B. B. Brandt and P. Majumdar, Phys. Lett. B 682 (2009) 253 [arXiv:0905.4195 [hep-lat]].

[17] A. Mykkänen, JHEP 1212 (2012) 069 [arXiv:1209.2372 [hep-lat]].

[18] M. Teper, Acta Phys. Polon. B 40 (2009) 3249 [arXiv:0912.3339 [hep-lat]].

[19] A. Athenodorou, B. Bringoltz and M. Teper, JHEP 1102 (2011) 030 [arXiv:1007.4720 [hep-lat]].

[20] A. Athenodorou, B. Bringoltz and M. Teper, JHEP 1105 (2011) 042 [arXiv:1103.5854 [hep-lat]].

[21] M. Pepe, PoS LATTICE 2010 (2010) 017 [arXiv:1011.0056 [hep-lat]].

[22] B. B. Brandt, JHEP 1102 (2011) 040 [arXiv:1010.3625 [hep-lat]].

[23] M. Billo et al, JHEP 1205 (2012) 130 [arXiv:1202.1984 [hep-th]].

[24] P. Bialas et al, Nucl. Phys. B 836 (2010) 91 [arXiv:0912.0206 [hep-lat]].

[25] M. Caselle et al, JHEP 1104 (2011) 020 [arXiv:1102.0723 [hep-lat]].

[26] M. Caselle et al, JHEP 0709 (2007) 117 [arXiv:0707.0055 [hep-lat]].

[27] M. Lüscher and P. Weisz, JHEP 0109 (2001) 010 [hep-lat/0108014].

[28] S. Dalley, Phys. Rev. D 74 (2006) 014025 [hep-th/0512264].

[29] B. Lucini and M. Panero, Phys. Rept. 526 (2013) 93 [arXiv:1210.4997 [hep-th]]. 